\title{
Integration of low-cost, dry-comb EEG-electrodes with a standard electrode cap for multimodal signal acquisition during human experiments
}

\author{
Christian Kuster Erichsen $^{1}$, Henrikke Dybvik², Martin Steinert ${ }^{3}$ \\ ${ }^{1}$ The Norwegian University of Science and Technology (NTNU), Norway \\ cnerich@stud.ntnu.no \\ ${ }^{2}$ The Norwegian University of Science and Technology (NTNU), Norway \\ henrikke.dybvik@ntnu.no \\ ${ }^{3}$ The Norwegian University of Science and Technology (NTNU), Norway \\ martin.steinert@ntnu.no
}

\begin{abstract}
This paper describes the development of a convenient and fast integration of low-cost, springloaded, dry electroencephalography (EEG) electrodes with a research-grade sensor cap ensuring electrode positioning according to the five percent system. Measuring brain activity is of increasing interest in fields outside of psychology and neuroscience, such as engineering. Human error often occurs due to lapses of attention, an inability to fully understand consequences or inadequacies in interface design. Effective design solutions incorporating and recognizing human behavior and various types of responses are needed to mitigate human error. Physiology sensors can be used to better evaluate which design meets user needs in the best way. Brain activity sensors have been applied within the brain-computer interface (BCI) community for decades. EEG is a highly popular modality due to its non-invasive nature and high temporal resolution. Prior research demonstrates increased performance of experimental results when using multimodal measurements compared to unimodal in prediction and classification tasks. Thus, we wanted to integrate EEG with an existing experimental setup which included functional Near-Infrared Spectroscopy (fNIRS). An integration was developed by means of rapid prototypes in cycles of design-build-test. The proposed setup increases available electrode positions, compared to currently available low-cost equipment, and constitutes a pragmatic, low-cost approach for integrating EEG-measurements in combination with other brain activity sensors, such as fNIRS. A proof-of-concept test of the signal quality was performed by means of two tasks shown to reveal easily detectable changes in the EEGsignal: closing the eyes and eye-blinking. Closing the eyes gave an increase in peak amplitude in the alpha range, an effect that was reversed once the eyes were opened. Deliberately blinking the eyes in specific intervals produced characteristic electrooculographic (EOG) artifacts in the signal. Both responses agree with the literature. The proposed solution aims to lower the barrier to including EEG as an additional modality in existing experimental setups, and thus increase the performance of experimental results.
\end{abstract}

Keywords: EEG, fNIRS, prototyping, human-centered design, experimentation 


\section{Introduction}

\subsection{Background and motivation}

\subsubsection{Affective engineering and its importance to interface design}

Measuring brain activity is becoming of increasing relevance in fields outside of psychology and neuroscience, such as engineering. The use of sensors that measure brain activity, especially electroencephalography (EEG), has been widely applied within the brain-computer interface (BCI) community for decades, see for instance Vidal (1973). The BCI community concerns itself with technology that enables control of computers and machines through commands generated by cognitive activity in the brain. However, the emotional response, also referred to as affective response, in humans when interacting with technological systems should be of obvious interest for a wider range of professionals, including product developers and interaction designers. Accident statistics suggest that a majority of accidents in a wide range of industries are caused by human error (Hansen, 2007; Wiegmann \& Shappell, 2017). Human error often occurs due to lapses of attention, an inability to understand the full consequence of an accident, or inadequacies in interface design. Effective design solutions that incorporate and recognizes human behavior and various types of responses are needed to mitigate human error. The increased awareness of the importance of the human response in interaction with technical systems has led to the call for integrating human affect when designing human-machine interactions (HMI) (Balters \& Steinert, 2017). A multitude of physiology sensors can be used to better evaluate which product solution or interface design meets user needs in the best way (Balters \& Steinert, 2014, 2017).

\subsubsection{Increasing hardware availability}

With the increased interest and research on BCI, there has been an improvement in the cost and accessibility of the necessary hardware to measure brain activity. EEG is a highly popular modality due to its non-invasive nature and high temporal resolution. Readily available, lowcost, consumer-grade EEG systems from several suppliers such as Emotiv, NeuroSky, and OpenBCI are being applied in a wide range of research today (Sawangjai, Hompoonsup, Leelaarporn, Kongwudhikunakorn, \& Wilaiprasitporn, 2019). Although these devices do not possess the same certifications as the medical-grade equipment, studies have shown that they can be comparable in terms of quality (Frey, 2016). Frey (2016) compared the consumer-grade OpenBCI Cyton with the medical-grade g.USBamp EEG amplifier, and found almost identical performance in the P300 speller task and workload monitoring during the n-back task.

\subsubsection{Advantages of multimodal data-capture}

EEG is a neuroimaging tool measuring electrical brain activity, notably the local current flow caused by neural activation. The sum of all activity of neurons in close vicinity constitutes an EEG signal, which is measured by two or more electrodes (Balters \& Steinert, 2017). Functional Near-Infrared Spectroscopy (fNIRS) is another neuroimaging technique that optically measures the hemodynamic response in brain tissue, i.e. concentration changes of oxygenated and deoxygenated hemoglobin following neural activation (Pinti et al., 2018). Two or more optodes, i.e. a minimum of one source and one detector must be used to measure cerebral blood flow. EEG has a high temporal resolution (in the millisecond range) and low spatial resolution (Balters \& Steinert, 2017; Pinti et al., 2018). EEG is also susceptible to noise (Al-Shargie et al., 2016). fNIRS offers better spatial resolution (Al-Shargie et al., 2016; Pinti et al., 2018), is more robust to motion, but has lower temporal resolution, in addition to a $\sim 5$ second delay from stimuli onset to response peak due to the nature of the hemodynamic response (Pinti et al., 2018). Both EEG and fNIRS offer several advantages; they are non-invasive, portable, less 
expensive than other brain-imaging techniques (such as MRI and PET) and safe for longer-term monitoring (Al-Shargie et al., 2016; Pinti et al., 2018). They have been reported to be a good combination (Al-Shargie et al., 2016). Prior research has found that multimodal signal analysis can improve performance in predicting human reactivity (Cisler, Greenwood, Roberts, McKendrick, \& Baldwin, 2019) and signal classification (Fazli et al., 2012; Lee, Fazli, Mehnert, \& Lee, 2015). Fazi et al. (2012) found that combining fNIRS with EEG data significantly increased the accuracy in classifying visualized movements. Al-Shargie et al. (2016) found EEG and fNIRS measurements to improve the classification accuracy of mental stress, compared to EEG only and fNIRS only.

Multimodal integration of EEG and fNIRS is still relatively new (Ahn \& Jun, 2017), which means there are limited options for simple multimodal data capture or integration. Current EEGfNIRS integrations include; custom built fNIRS-system to fit an existing EEG-system with the need for two desktop computers for simultaneous data recording (Ahn, Nguyen, Jang, Kim, \& Jun, 2016), or needing to purchase two separate systems, record data independently and having to fuse the data post-experiment (Al-Shargie et al., 2016; Fazli et al., 2012).

\subsection{Objective and scope of the paper}

Based on existing access to one brain activity sensor, namely fNIRS, we wanted to integrate EEG measurements to improve experimental results. Thus, we wanted a low-cost EEG integration to an existing fNIRS system to enable multimodal measurements during HMI experiments. It should be compatible with a standard electrode cap and optode placement, with the ability to quickly adapt to new setups, including different caps and sensor montages.

This paper presents the result, a functional prototype of an EEG integration. A low-cost, adaptive integration of dry-comb EEG-electrodes in a standard electrode cap, fitted with fNIRS optodes, has been developed. This paper demonstrates the development process, the final prototype and initial tests demonstrating a proof-of-concept. Following this introduction is a section describing the hardware basis used in the following development process. The methodology, the development process, and the test protocol are described in section three. Section four presents the resulting prototype, both the EEG adaption and system integration before showing the results of the proof-of-concept tests. Discussion and conclusion follow.

\section{Hardware spesifications}

The paper covers the integration of the Cyton biosensing board (OpenBCI Inc., 2019) in combination with their spring-loaded, dry EEG electrodes (Figure 1) provided as part of the Ultracortex Mark IV EEG headset (OpenBCI Inc., 2019). OpenBCI provides three different dry electrodes: flat, spikey or $5 \mathrm{~mm}$ combs respectively. The developed adapters are compatible with all the available dry electrodes OpenBCI provides. Among the low-cost EEG-systems available, OpenBCI was chosen due to minimalistic form factor, low weight, small size, and open-source nature, lending itself to be modified and hacked to meet the researchers' requirements. The performance of the Cyton board has also been found to be comparable to medical-grade EEG-amplifiers (Frey, 2016), which makes it an interesting option for nonclinical research. The key specifications of the OpenBCI Cyton are listed in Table 1. 
Table 1. Key specifications OpenBCI Cyton

\begin{tabular}{|l|l|}
\hline Channels & 8 \\
\hline Compatible electrodes & Active \& Passive \\
\hline Data resolution & 24-bit \\
\hline Programmable gain & $1,2,4,6,8,12,24$ \\
\hline Operating voltage & $3.3 \mathrm{~V}$ Digital / +/- 2.5V analog \\
\hline Amplifier & Texas Instruments ADS1299 ADC \\
\hline Microcontroller & PIC32MX250F128B \\
\hline
\end{tabular}

OpenBCI provides spring-loaded, dry electrodes intended for use with their 3D-printed headset. Although this system might be well suited for simple, low-cost EEG applications, it has several drawbacks rendering it impractical for more elaborate experiments. Most notably, the headset only accommodates 35 electrode positions and does not lend itself to be used in combination with other sensors such as fNIRS. Thus, the authors needed to develop a set of custom adapters to integrate the EEG dry electrodes with a standard fNIRS cap. The goal was to integrate the spring-loaded dry EEG electrodes (Figure 1) provided by OpenBCI Inc. into a standard electrode cap, namely the EASYCAP AC-128-X1-C-58, which has a 128-channel layout according to the five percent system (Oostenveld \& Praamstra, 2001). This system is a part of the international EEG system, a standardized method for consistent description of electrode placement on the scalp, to enable greater experiment replicability.

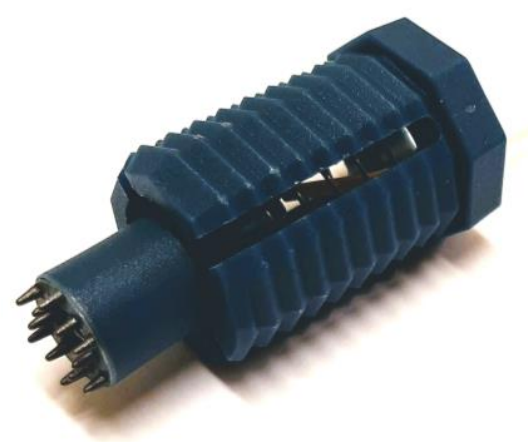

Figure 1. Spring-loaded dry electrode from OpenBCI.

\section{Method and development}

\subsection{Development methodology}

The guiding development methodology was Wayfaring (Steinert \& Leifer, 2012). Rapid prototypes in cycles of design-build-test (Gerstenberg, Sjöman, Reime, Abrahamsson, \& Steinert, 2015; Leikanger, Balters, \& Steinert, 2016) were made based on initial hardware specifications and requirements for EEG integration. Design-build-test cycles are argued to be effective for problem-solving in product development projects (Wheelwright \& Clark, 1994), suited for a dynamic environment (Gerstenberg et al., 2015). 


\subsection{Development process}

The development process consisted of rapid iterations of several prototypes during designbuild-test cycles. Since the product was small in size, prototypes could be produced quickly through 3D-printing. All prototypes were printed on a consumer-grade desktop 3D-printer, with the parameters specified in Table 2.

Table 2. Printing parameters.

\begin{tabular}{|l|l|}
\hline Printer & Prusa MK3 \\
\hline Material & PLA \\
\hline Nozzle diameter & $0.4 \mathrm{~mm}$ \\
\hline Extruder temperature & $210^{\circ} \mathrm{C}$ (first layer:215 $\left.{ }^{\circ} \mathrm{C}\right)$ \\
\hline Bed temperature & $60{ }^{\circ} \mathrm{C}$ \\
\hline Layer height & $0.15 \mathrm{~mm}$ \\
\hline Perimeters vertical shells & 2 \\
\hline Solid layers top & 7 \\
\hline Solid layers bottom & 5 \\
\hline
\end{tabular}

\subsubsection{Iteration 1 - Mounting}

A simple prototype with circular geometry was made based on manual measurements of the electrode, electrode housing and the optode-mounts that were supplied with the cap originally. The slots enabling the electrode to slide back and forth in the housing also make the housing flexible (Figure 1). The first prototype (Figure 2) was designed to test whether or not a simple snap-lock mechanism would be feasible due to the flexible properties of the electrode housing.
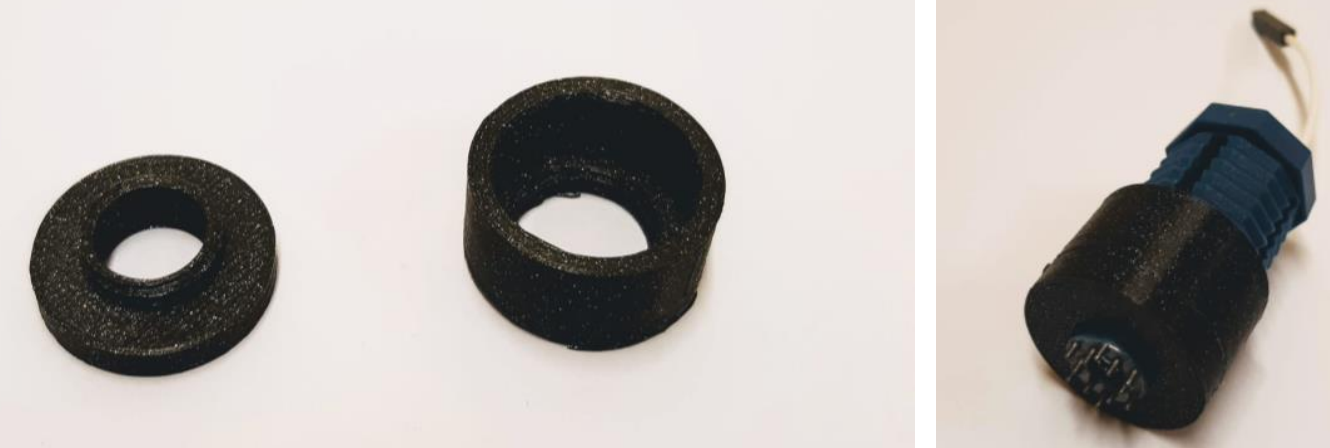

Figure 2. First iteration prototype. Bottom and top part of the adapter (left). Assembled adapter attached to the dry electrode (right).

Initial testing of the prototype showed that the snap-lock did not provide enough grip on the electrode housing when wearing the cap. The threads on the electrode housing also made the electrodes prone to tilting when interacting with the adapter. Additionally, the outer diameter of the bottom (scalp-facing) part was found to be too large, causing poor flexibility in the cap, if mounted closely together.

\subsubsection{Iteration 2 - Threaded mount}

Addressing the issues discovered by the first prototype, a threaded connection between the adapter and the electrode housing was implemented. The threads on the housing are not standard threads, which complicate precision in modeling. However, due to the open-source nature of the OpenBCI products, STL-files of all components of the Ultracortex Mark IV headset are freely available under the GNU General Public License on Github (OpenBCI/Ultracortex, 2015/2020). By importing the geometry of the threads into the design of the adapters, a good connection between the dry electrodes and the adapters was achieved. 
The diameter of the bottom (scalp-facing) part of the adapter was also reduced to improve flexibility in the cap when several adapters are mounted in close proximity.

\subsubsection{Iteration 3 - Improving handling}

To improve the handling of the adapters during assembly and ensure better fit with the electrode housing, outer geometry was made octagonal. This made connecting the top and bottom part of the adapter easier when installing in the cap. As a secondary benefit, it reduced the tendency of the electrode housing to unscrew itself when mounting the cap on test-subjects. A 3D model of the final design is illustrated in Figure 3, while Figure 7 depicts the exterior and interior view of a fully mounted electrode.
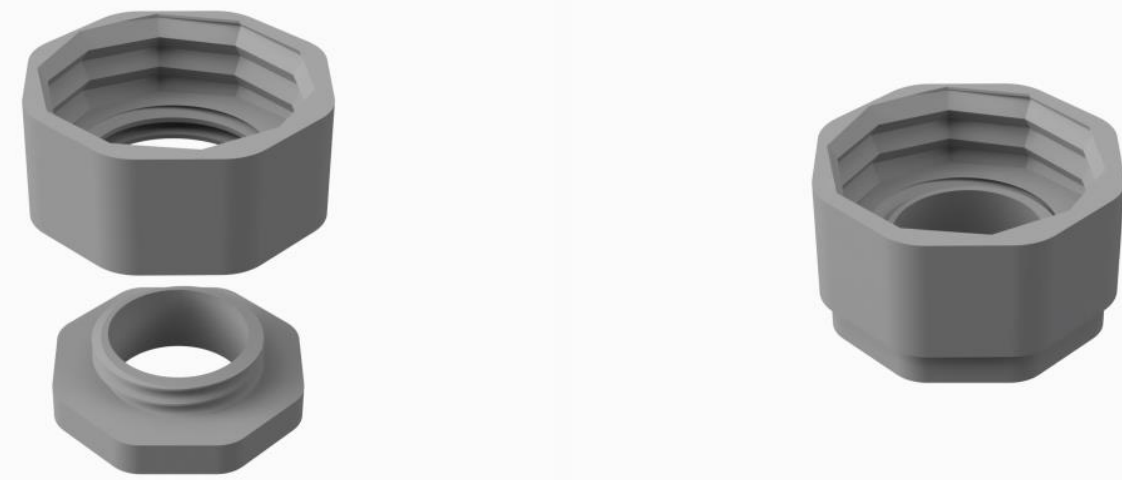

Figure 3. 3D-model of the final prototype. Exploded view (left) and assembled (right).

\subsubsection{Mounting the Cyton board and battery pack}

As the final step in development, fixtures to mount the Cyton board and the battery pack to the cap were prototyped, see Figure 4 and Figure 5. Although the board and battery pack does not necessarily have to be mounted on the subjects head, we deemed it beneficial to mount the Cyton board close to the electrodes. Placing the Cyton board elsewhere would require a longer wire, increasing electrical resistance in the system and making it more vulnerable to electrical noise. Furthermore, placing the board and battery on the subject's head imposes fewer restrictions on the subject's seating position or movement. This is advantageous for in-situ experiment applications. To fixate them to the cap, the existing fNIRS optode holders were exploited. Due to the low weight of the Cyton board and battery pack, a simple cylindrical feature with a tight-fit was sufficient to keep the components in place during stationary testing. The mounts can be assembled by means of screws, glue, or as demonstrated in Figure 4, with standard rubber bands. Due to the weight of the battery pack, the placement should be close to the top of the head (position $\mathrm{Cz}$ ), to reduce the risk of inducing movement of the cap. Alternatively, a light-weight battery pack can be used. If this is not feasible with the desired

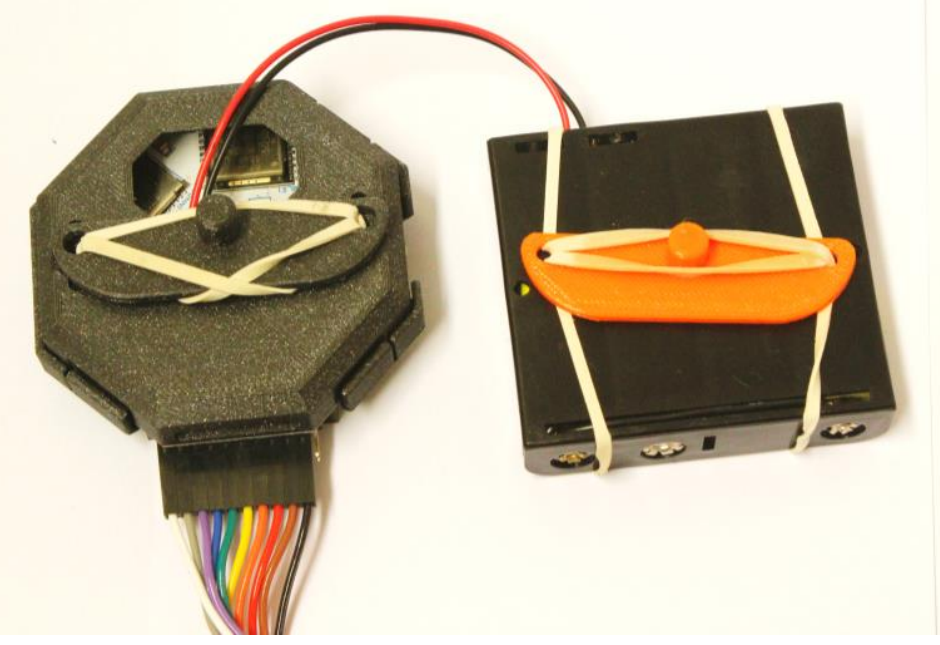


electrode montage, we propose using a battery pack with longer wires that enable fixating the battery pack to the subject's body instead.

Figure 4. Mounting fixture attached to the battery pack and Cyton board with housing, by means of rubber bands.

\subsection{Testing signal quality - protocol for a proof-of-concept test}

As a preliminary test of the signal quality, we performed two tasks that have been shown to reveal easily detectable changes in the EEG-signal: closing the eyes, and eye-blinking. Since formal EEG analysis is not within the scope of this paper, we relied on the output from the OpenBCI GUI software for these initial tests. EEG signals are often separated in distinct frequency bands during analysis (i.e. Delta (1-4 Hz), Theta (4-8 Hz), Alpha (8-13 Hz) and Beta $(13-30 \mathrm{~Hz})$ ) and used to examine emotional states (Al-Shargie et al., 2016). Signal artifacts can be identified through visual inspection (Krishnaveni, Jayaraman, Aravind, Hariharasudhan, \& Ramadoss, 2006) and used to assess if the EEG signal is correct. Three OpenBCI dry-electrodes were mounted in position Fp1 (channel 1), Fp2 (channel 2), and O2 (channel 8), while the reference electrodes were mounted in position A1, and A2. The locations Fp1 and Fp2 cover the prefrontal cortex, while position $\mathrm{O} 2$ covers the visual cortex. Positions A1 and A2 are situated on the left and right earlobe respectively (Figure 5).
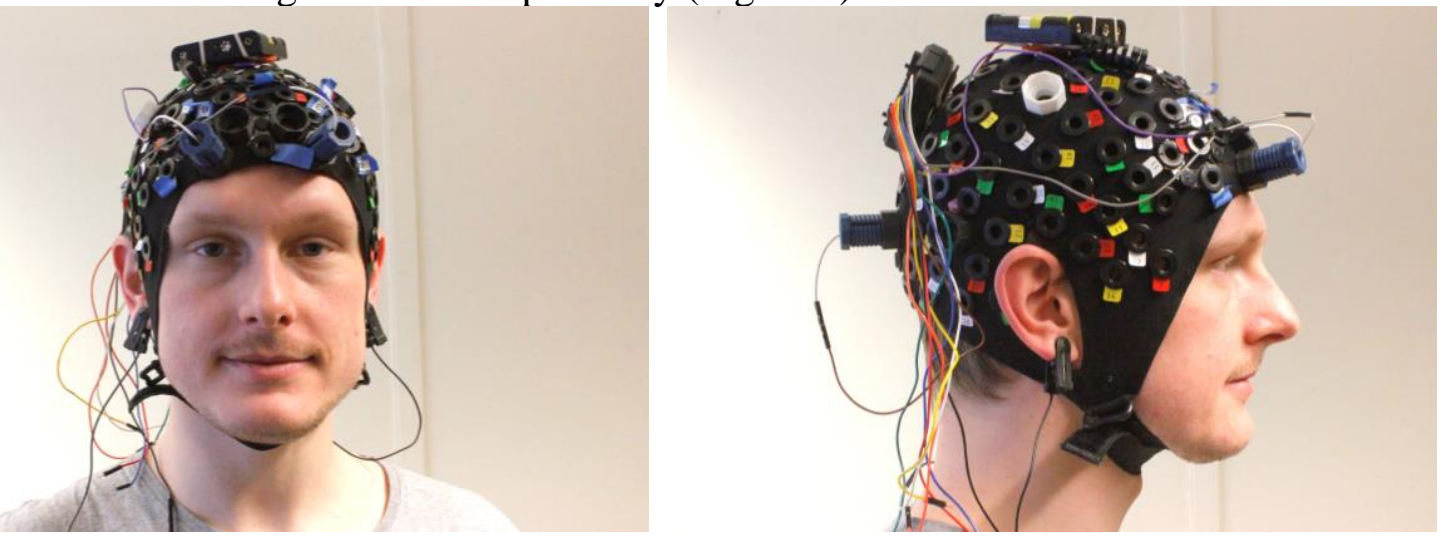

Figure 5. Setup during initial testing.

\subsubsection{Induce increase in the alpha range}

According to Mulholland (1995), closing the eyes should cause a visual increase in the alpha range $(8-13 \mathrm{~Hz})$ amplitude. Thus, we measured the EEG response with open eyes, closed eyes, and then after opening the eyes again. The data was captured and displayed by the OpenBCI GUI software (OpenBCI GUI, 2019) and visually inspected. The purpose of this test was to provide proof-of-concept.

\subsubsection{Produce ocular artifacts}

Electrooculographic (EOG) artifacts, also referred to as ocular artifacts (OA), are one of the most common and well-known disturbances in EEG signals (Krishnaveni et al., 2006; Vidal, 1973; Zeng, Song, Yan, \& Qin, 2013). EOG artifacts are caused by interference from the electrical field that is induced when the eyeballs rotate in their sockets. Since these electrical potentials are very large compared to brain induced potentials, failure to identify EOGs would imply unacceptable signal quality. A simple test was conducted to verify the ability to detect EOGs. The subject wearing the headset would deliberately blink their eyes in intervals of roughly five seconds. Between blinks, efforts were made to restrain from blinking and movement of the eyes. Subsequent inspection of the signal response displayed in the time-series plot in the OpenBCI GUI was performed. 


\section{Results}

This section first describes the proposed setup, e.i, the final prototype. An initial test was made as a proof-of-concept to ensure the final prototype functioned as intended. The results of these tests are described in section 4.2.

\subsection{Resulting prototype}

\subsubsection{EEG integration}

The final prototype consists of two octagonal parts with a circular, central hole for insertion of the EEG electrodes (Figure 3); The bottom part (height $=3 \mathrm{~mm}$, outer diameter $=21.6 \mathrm{~mm}$, inner diameter $=11.2 \mathrm{~mm}$ ) is mounted on the inside of the cap, extending through the pre-cut holes in the cap and connected to the top part by means of a threaded connection (Figure 6). The top part (height $=9.35 \mathrm{~mm}$, outer diameter $=25.6 \mathrm{~mm}$ ) is threaded to allow fast fixation of the spring-loaded dry electrodes. Figure 7 illustrates a fully assembled adapter with a mounted electrode. The adapters can be mounted on any of the precut holes in the cap. The setup has been tested with EASYCAP AC-128-X1-C-58 (see Figure 6). The costs associated with the setup (excluding fNIRS device and cap) is limited to $\$ 849$ for the hardware supplied by OpenBCI (excluding shipping), and roughly $\$ 1$ in filament cost, assuming free access to a 3Dprinter.

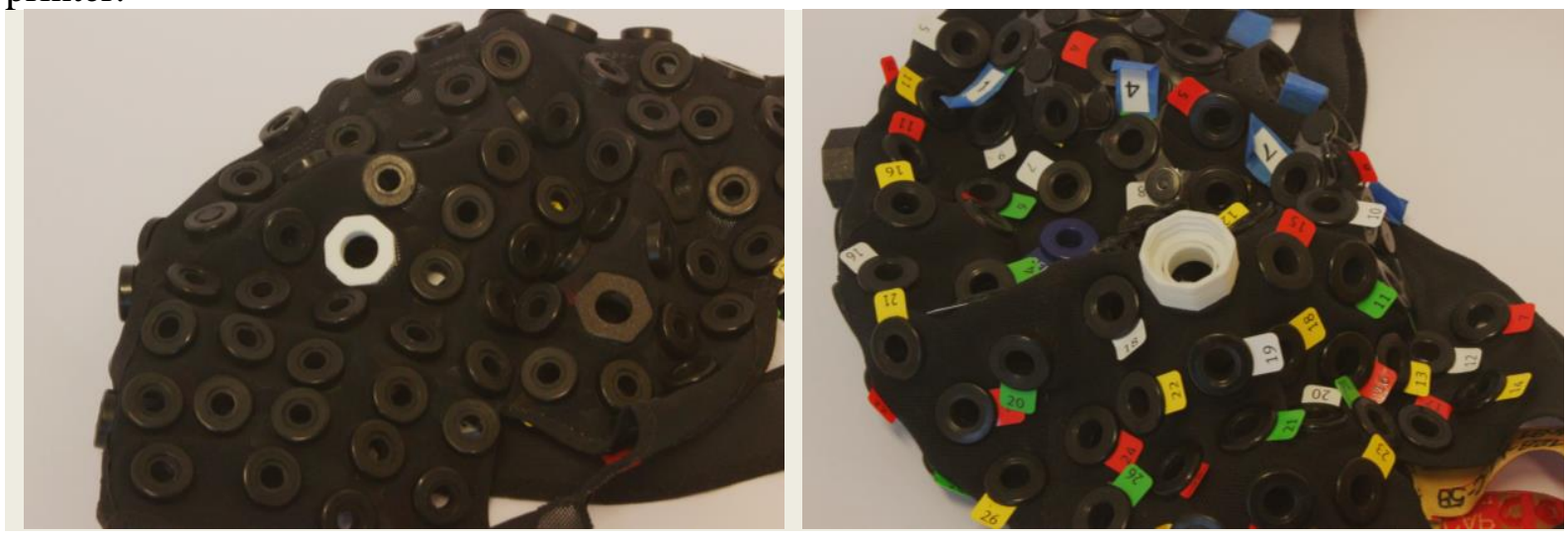

Figure 6. Mounted adapter, interior view (left), and exterior view (right).
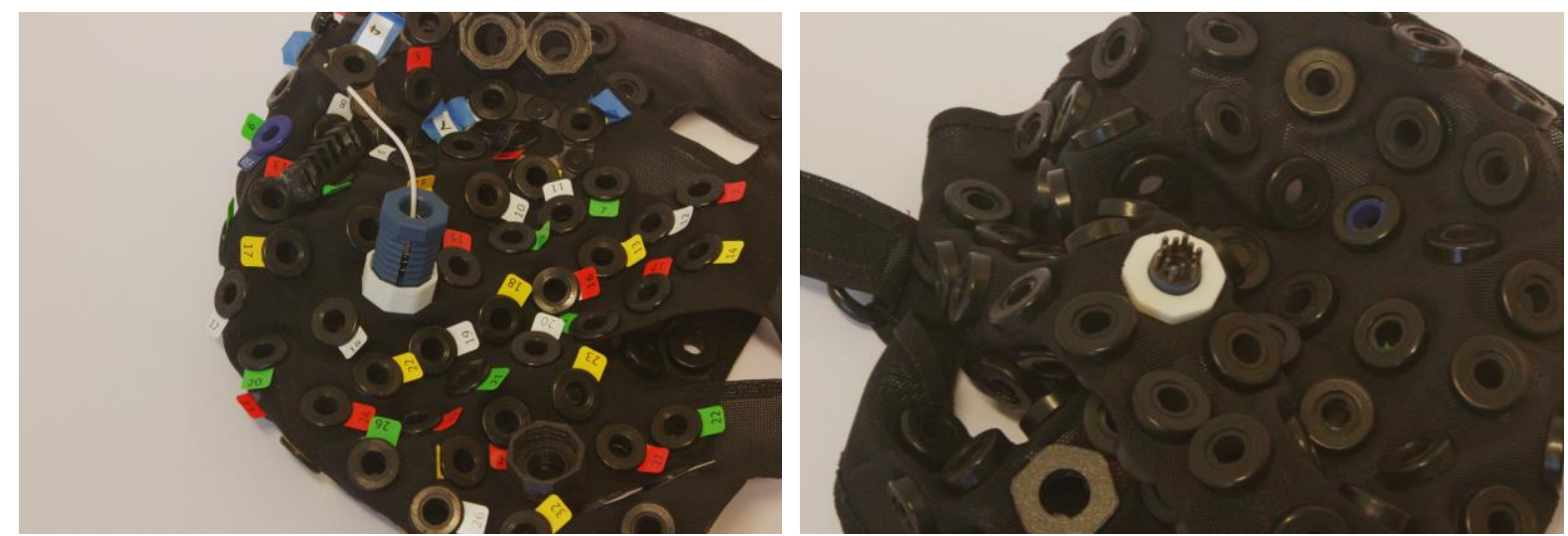

Figure 7. Dry EEG electrode mounted. Exterior view (left), interior view (right).

\subsubsection{Simultaneous recording of fNIRS and EEG}

Figure 8 shows the final setup including fNIRS. A pilot experiment utilizing the setup is currently being conducted which intends to verify the ability to record high-quality data of both modalities simultaneously. This experiment nor its data analysis is in the scope of this paper, so reservations must be made regarding the signal quality of the final setup. However, the 
results from the test described in section 4.2 indicate the feasibility of acquiring acceptable measurements.

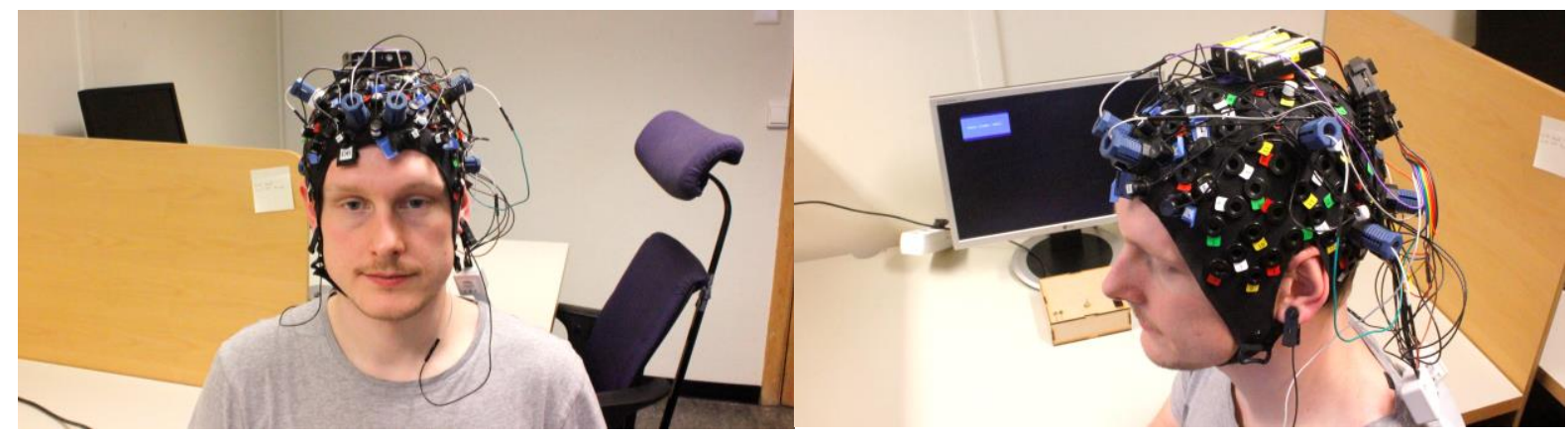

Figure 8. Eight channels EEG integrated and 20/40 channel fNIRS fully integrated.

\subsubsection{Software integration}

One of the advantages of the OpenBCI platform is that the raw data is readily available and accessible. If only EEG-recordings are of interest, raw data can be recorded with the free OpenBCI GUI application. For multimodal measurements, raw data can also easily be streamed using lab streaming layer, which enables synchronization of multiple sensors and forwarding to third-party software, see Figure 9. The proposed setup was tested by streaming EEG and fNIRS data simultaneously to iMotions (IMotions, 2020) successfully.

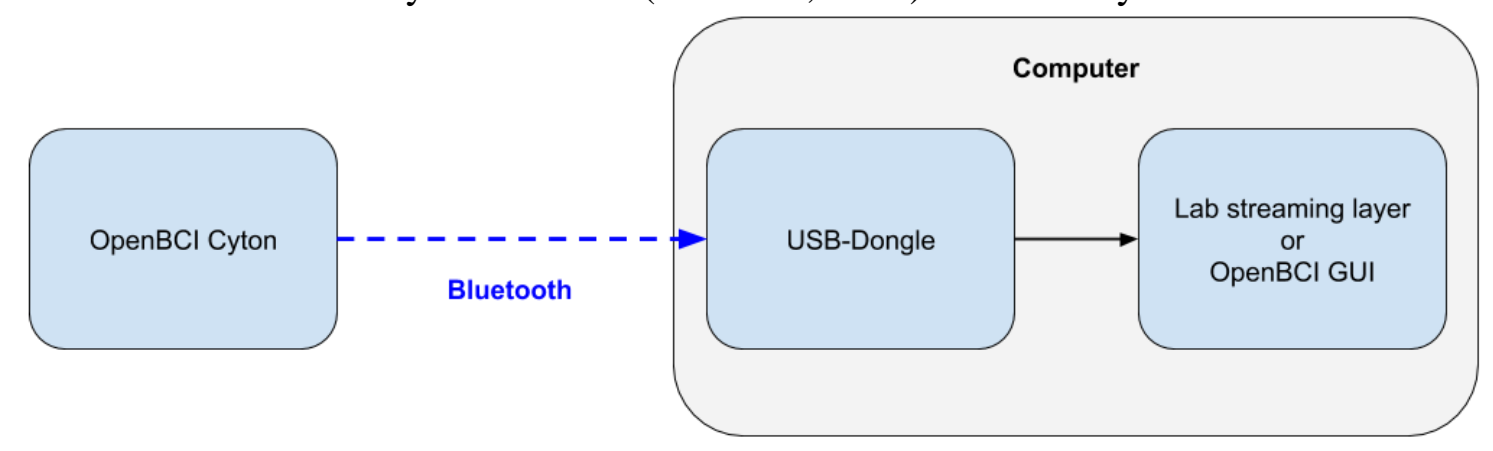

Figure 9. Schematic overview of raw data flow.

\subsection{Proof-of-concept test}

The results described in this section are from the initial tests made with EEG-electrodes integrated into EASYCAP using the final prototype, measuring EEG only.

\subsubsection{Eyes closed}
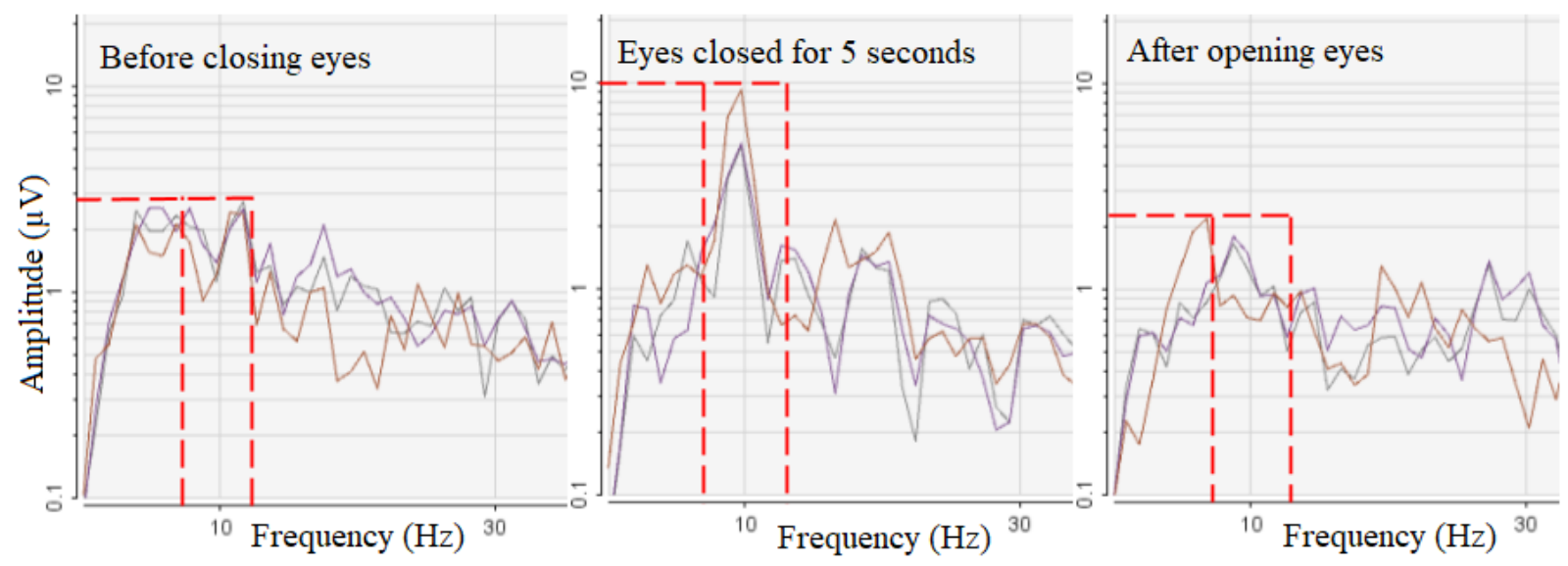

Figure 10. OpenBCI GUI. 
Figure 10 shows the power spectrum (EEG-response) prior to closing the eyes, approximately five seconds after closing the eyes, and shortly after opening the eyes again. This clearly indicates that the peak amplitude in the alpha range increase during the period with eyes closed. Furthermore, this effect is reversed once the eyes are opened again. This response was as expected and in accordance with Mulholland (1995).

\subsubsection{Eye blinking}

Figure 11 shows the results of the test of controlled eye-blinking. The time-series plot of the recorded signals displays characteristic artifacts in agreement with what is reported in the literature (Krishnaveni et al., 2006; Zeng et al., 2013).

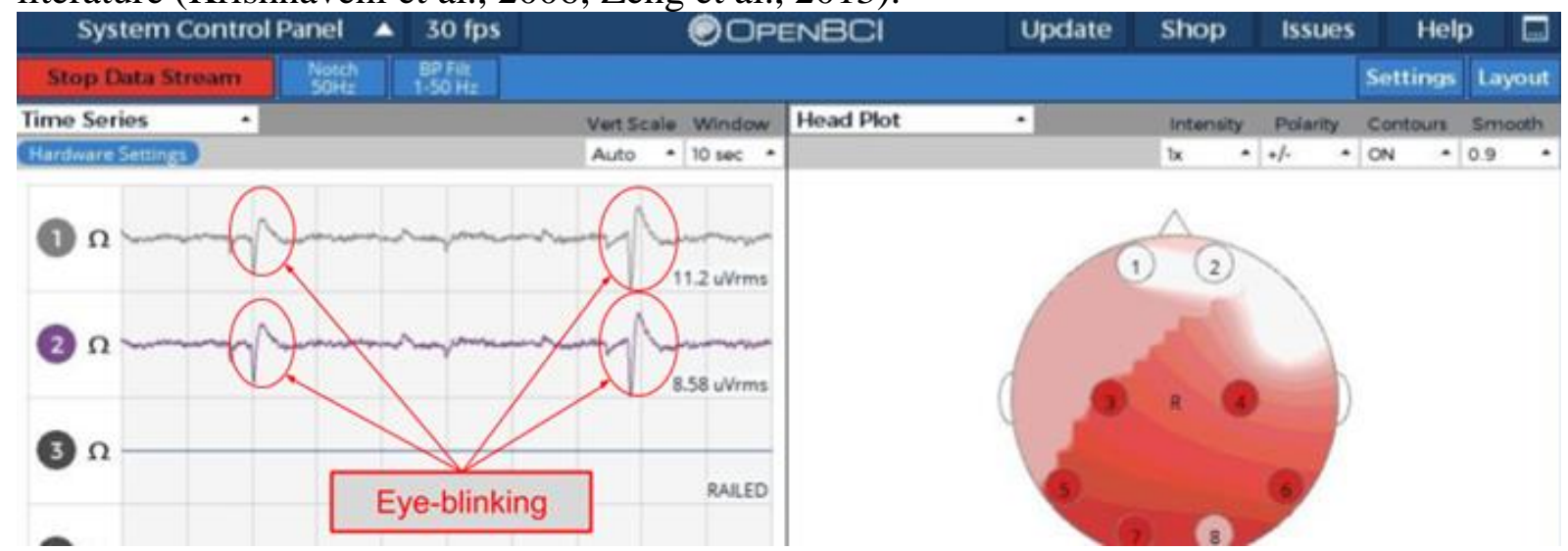

Figure 11. OpenBCI GUI. EOGs clearly visible in channels one and two, positioned at Fp1 and Fp2 respectively.

\section{Discussion}

When used exclusively with EEG electrodes, the ability to detect EOGs and an increase in alpha-range frequencies was demonstrated using the developed EEG electrode integration. The results are in accordance with the expected behavior (Mulholland, 1995; Vidal, 1973; Zeng et al., 2013). The purpose of these tests was to provide proof-of-concept. Although the tests lack scientific rigor, it indicates that a minimal acceptable performance is achieved. Evaluation of electrode performance is a complicated issue in its own regard (Lopez-Gordo, Sanchez-Morillo, \& Valle, 2014; Tăuţan, Serdijn, Mihajlović, Grundlehner, \& Penders, 2013) and is considered beyond the scope of this paper. A larger experiment utilizing both modalities is currently being piloted to ensure sufficient data quality and system performance. Initial results are promising, however not within the scope of this paper. The setup has only been tested with EASYCAP AC-128-X1-C-58. The proposed solution should be applicable to other soft-fabric sensor caps. However, small modifications might be necessary. During preliminary testing of the setup on multiple subjects some challenges acquiring acceptable signals were encountered. This might be explained by differences in hair-thickness and general skin conductance. Skin conductance properties are highly individual, in addition to that some individuals do not exhibit sufficient levels of electrodermal activity for good measurements. Since the electrodes require skin contact, acquiring high-quality EEG-signals in hair-covered regions remains an inherent challenge and might require additional efforts in some cases. A conductive gel can be applied to the electrodes to better secure skin contact.

\section{Conclusion}

This paper describes the design and development of an adaptive EEG integration to a researchgrade sensor cap ensuring electrode positioning according to the five percent system. The proposed setup enables a low-cost solution to supplement fNIRS-measurements with EEG data. 
The proposed setup enables convenient and fast integration of low-cost, spring-loaded, dry electrodes, and it increases the available electrode positions compared to currently available equipment. It constitutes a pragmatic approach for integrating EEG-measurements with other brain activity sensors and requires minimal investment. The proposed solution aims to lower the barrier to include EEG as an additional modality in existing experimental setups, which can increase the quality of experimental results.

\section{Acknowledgment}

This research is supported by strategic funds from the department for Mechanical and Industrial Engineering (MTP) at NTNU.

\section{References}

Ahn, S., \& Jun, S. C. (2017). Multi-Modal Integration of EEG-fNIRS for Brain-Computer Interfaces - Current Limitations and Future Directions. Frontiers in Human Neuroscience, 11. https://doi.org/10.3389/fnhum.2017.00503

Ahn, S., Nguyen, T., Jang, H., Kim, J. G., \& Jun, S. C. (2016). Exploring Neuro-Physiological Correlates of Drivers' Mental Fatigue Caused by Sleep Deprivation Using Simultaneous EEG, ECG, and fNIRS Data. Frontiers in Human Neuroscience, 10. https://doi.org/10.3389/fnhum.2016.00219

Al-Shargie, F., Kiguchi, M., Badruddin, N., Dass, S. C., Hani, A. F. M., \& Tang, T. B. (2016). Mental stress assessment using simultaneous measurement of EEG and fNIRS. Biomedical Optics Express, 7(10), 3882. https://doi.org/10.1364/BOE.7.003882

Balters, S., \& Steinert, M. (2014). Decision-making in engineering-a call for affective engineering dimensions in applied engineering design and design sciences. 11-15. IEEE.

Balters, S., \& Steinert, M. (2017). Capturing emotion reactivity through physiology measurement as a foundation for affective engineering in engineering design science and engineering practices. Journal of Intelligent Manufacturing, 28(7), 1585-1607. https://doi.org/10.1007/s10845-015-1145-2

Cisler, D. S., Greenwood, P. M., Roberts, D. M., McKendrick, R., \& Baldwin, C. L. (2019). Comparing the Relative Strengths of EEG and Low-Cost Physiological Devices in Modeling Attention Allocation in Semiautonomous Vehicles. Front. Hum. Neurosci. https://doi.org/10.3389/fnhum.2019.00109

Fazli, S., Mehnert, J., Steinbrink, J., Curio, G., Villringer, A., Müller, K.-R., \& Blankertz, B. (2012). Enhanced performance by a hybrid NIRS-EEG brain computer interface. NeuroImage, 59(1), 519-529. https://doi.org/10.1016/j.neuroimage.2011.07.084

Frey, J. (2016). Comparison of an open-hardware electroencephalography amplifier with medical grade device in brain-computer interface applications. ArXiv:1606.02438 [Cs]. Retrieved from http://arxiv.org/abs/1606.02438

Gerstenberg, A., Sjöman, H., Reime, T., Abrahamsson, P., \& Steinert, M. (2015). A Simultaneous, Multidisciplinary Development and Design Journey - Reflections on Prototyping. Entertainment Computing - ICEC 2015, 409-416. https://doi.org/10.1007/978-3-319-24589-8_33

Hansen, F. D. (2007). Human Error: A Concept Analysis. Journal of Air Transportation, 11(3), 61-77.

IMotions (Version 8.1). (2020). Retrieved from https://imotions.com/

Krishnaveni, V., Jayaraman, S., Aravind, S., Hariharasudhan, V., \& Ramadoss, K. (2006). Automatic Identification and Removal of Ocular Artifacts from EEG using Wavelet Transform. MEASUREMENT SCIENCE REVIEW, 6(4), 13.

Lee, M.-H., Fazli, S., Mehnert, J., \& Lee, S.-W. (2015). Subject-dependent classification for robust idle state detection using multi-modal neuroimaging and data-fusion techniques 
in BCI. Pattern Recognition, 48(8), 2725-2737.

https://doi.org/10.1016/j.patcog.2015.03.010

Leikanger, K. K., Balters, S., \& Steinert, M. (2016). Introducing the wayfaring approach for the development of human experiments in interaction design and engineering design science. Presented at the DS 84: Proceedings of the DESIGN 2016 14th International Design Conference.

Lopez-Gordo, M. A., Sanchez-Morillo, D., \& Valle, F. P. (2014). Dry EEG Electrodes. Sensors (Basel, Switzerland), 14(7), 12847-12870. https://doi.org/10.3390/s140712847

Mulholland, T. (1995). Human EEG, behavioral stillness and biofeedback. International Journal of Psychophysiology, 19(3), 263-279. https://doi.org/10.1016/01678760(95)00019-O

Oostenveld, R., \& Praamstra, P. (2001). The five percent electrode system for high-resolution EEG and ERP measurements. Clinical Neurophysiology, 112(4), 713-719. https://doi.org/10.1016/S1388-2457(00)00527-7

OpenBCI GUI (Version 4.1.7-beta.2). (2019). Retrieved from https://github.com/OpenBCI/OpenBCI_GUI

OpenBCI Inc. (2019). Retrieved from https://openbci.com/

OpenBCI/Ultracortex. (2020). Retrieved from https://github.com/OpenBCI/Ultracortex (Original work published 2015)

Pinti, P., Tachtsidis, I., Hamilton, A., Hirsch, J., Aichelburg, C., Gilbert, S., \& Burgess, P. W. (2018). The present and future use of functional near-infrared spectroscopy (fNIRS) for cognitive neuroscience. Annals of the New York Academy of Sciences, n/a(n/a). https://doi.org/10.1111/nyas.13948

Sawangjai, P., Hompoonsup, S., Leelaarporn, P., Kongwudhikunakorn, S., \& Wilaiprasitporn, T. (2019). Consumer grade EEG Measuring Sensors as Research Tools: A Review. IEEE Sensors Journal, 1-1. https://doi.org/10.1109/JSEN.2019.2962874

Steinert, M., \& Leifer, L. J. (2012). "Finding One's Way": Re-Discovering a Hunter-Gatherer Model based on Wayfaring. International Journal of Engineering Education, 28(2), 251 .

Tăuţan, A.-M., Serdijn, W., Mihajlović, V., Grundlehner, B., \& Penders, J. (2013). Framework for evaluating EEG signal quality of dry electrode recordings. 2013 IEEE Biomedical Circuits and Systems Conference (BioCAS), 186-189. https://doi.org/10.1109/BioCAS.2013.6679670

Vidal, J. J. (1973). Toward Direct Brain-Computer Communication. Annual Review of Biophysics and $\quad$ Bioengineering, 157-180. https://doi.org/10.1146/annurev.bb.02.060173.001105

Wheelwright, S. C., \& Clark, K. B. (1994). Accelerating the Design-build-test Cycle for Effective Product Development. International Marketing Review, 11(1), 32-46. https://doi.org/10.1108/02651339410057509

Wiegmann, D. A., \& Shappell, S. A. (2017). A human error approach to aviation accident analysis: The human factors analysis and classification system. Routledge.

Zeng, H., Song, A., Yan, R., \& Qin, H. (2013). EOG Artifact Correction from EEG Recording Using Stationary Subspace Analysis and Empirical Mode Decomposition. Sensors (Basel, Switzerland), 13(11), 14839-14859. https://doi.org/10.3390/s131114839 